\title{
Análise numérica de cantoneiras enrijecidas de aço formadas a frio submetidas à compressão
}

\author{
Murillo Silva Rocha ${ }^{1 *}$ e Wanderson Fernando Maia ${ }^{2}$ \\ ${ }^{1}$ Engenheiro Civil, AMBAR, Alameda Sinlioku Tanaka, 202, Lotes 46/47, Parque \\ Ecotec Damha, CEP 13565-261, São Carlos, SP, Brasil, \\ e-mail: murillosilvarocha@gmail.com \\ 2 Professor, Departamento de Engenharia Civil, Universidade Federal de São \\ Carlos, Rodovia Washington Luís (SP-310), Km 235, CEP 13565-905, São Carlos, \\ SP, Brasil, e-mail: wfmaia@ufscar.br
}

\section{Numerical analysis of cold-formed steel lipped angles under compression}

\begin{abstract}
Resumo
Este trabalho analisa a resposta estrutural de cantoneiras enrijecidas de aço formadas a frio submetidas a compressão centrada e excêntrica, por meio de análise numérica não-linear via elementos finitos. Este tipo de cantoneira apresenta três modos críticos de instabilidade: (i) modo global de flexão, (ii) modo global de flexo-torção, ambos críticos para barras longas, e o (iii) modo local, que é crítico para barras de menor comprimento. Os resultados da compressão centrada via elementos finitos foram comparados com os resultados de análise experimental realizada por Young (2005), indicando valores relativamente próximos. As seções submetidas a compressão centrada e excêntrica foram comparadas aos resultados provenientes dos procedimentos normativos. A norma se mostrou conservadora em relação a barras mais esbeltas e contra a segurança para barras curtas para as seções aqui analisadas.
\end{abstract}

Palavras-chave: Estruturas de aço, perfis formados a frio, cantoneira enrijecida, compressão centrada, compressão excêntrica.

\begin{abstract}
This paper analyze the structural response of cold-formed steel lipped angles under axial and eccentric compression, by means of nonlinear finite element analysis. This type of angle presents three critical buckling modes: (i) global-flexural mode, (ii) global-flexural-torsional mode, both critics for high length members, and (iii) local mode, which is critical for short length members. The results of the finite element axial compression were compared with the results of the experimental analysis performed by Young (2005), indicating relatively close values. The members under axial and eccentric compression were compared with the results from the normative procedures. The standard was conservative in relation to slender members and against safety in relation to short length members fo the sections analyzed here.
\end{abstract}

Keywords: Steel structures, cold-formed steel, lipped angle, axial compression, eccentric compression. 


\section{Introdução}

Os perfis de aço formados a frio são elementos que apresentam elevada resistência mecânica, com um menor consumo de material, sendo o processo de fabricação mais simples e barato, promovendo reduções no custo. Eles estão sendo cada vez mais utilizados como barras de estruturas treliçadas, barras de contraventamento e elementos auxiliares em ligações. No entanto, por serem perfis fabricados com chapas de espessura reduzida, apresentam diferentes modos de instabilidade que não ocorrem em perfis laminados e soldados.

As cantoneiras enrijecidas de aço formadas a frio são um tipo de perfil que apresenta três modos críticos de instabilidade: (i) modo global de flexão, (ii) modo global de flexo-torção, ambos críticos para barras mais longas, e o (iii) modo local, dominante em barras de menor comprimento. Elas apresentam melhor desempenho estrutural que as cantoneiras simples, mas em vista do maior número de dobras para sua fabricação, é necessário um estudo comparativo de custo-benefício pelo projetista. Na Figura 1 são ilustradas as seções transversais da cantoneira simples e da cantoneira enrijecida.
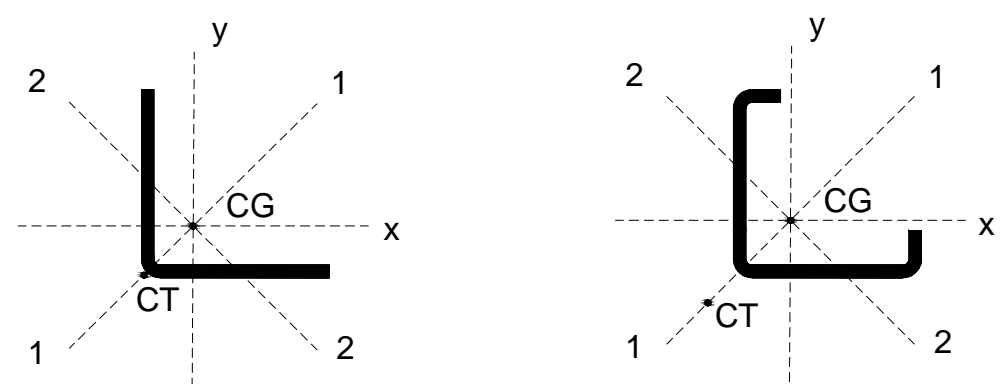

Figura 1 - Seção transversal: cantoneira simples e cantoneira enrijecida (Maia, 2008)

Apesar das normas apresentarem procedimentos para o dimensionamento de cantoneiras enrijecidas, ensaios experimentais e simulações numéricas computacionais realizados mostram que estas são excessivamente conservadoras, principalmente para barras com maior índice de esbeltez global. Este fato foi observado em trabalhos realizados por Young (2005), Shifferaw e Schafer (2011) e Shifferaw e Schafer (2014).

O principal objetivo do trabalho é investigar a resposta estrutural de cantoneiras enrijecidas submetidas à compressão centrada e excêntrica, por meio de análise numérica não-linear via elementos finitos, realizada no programa ANSYS. Os resultados obtidos são comparados com resultados de análise experimental obtidos por Young 
(2005). Além disso, são avaliados os resultados provenientes dos procedimentos normativos. Foram analisadas três diferentes seções, buscando estudar diferentes índices de esbeltez local e global, possibilitando assim, avaliar a influência de cada uma das variáveis envolvidas no problema.

\section{Previsões Normativas}

De acordo com a ABNT NBR 14762:2010, a força normal resistente de uma barra submetida à compressão centrada é calculada de acordo com a Equação (1).

$$
N_{c, R}=\chi \cdot A_{e f} \cdot f_{y}
$$

$\chi$ é o fator de redução associado a resistência à compressão, calculado de acordo com a Equação (2), $A_{e f}$ é a área efetiva da seção transversal, $f_{y}$ é a resistência ao escoamento do aço, $\lambda_{0}$ é índice de esbeltez reduzido, calculado de acordo com a Equação (3).

$$
\begin{aligned}
& \chi= \begin{cases}\left(0,658^{\lambda_{0}^{2}}\right) & \text { Para } \lambda_{0} \leq 1,5 \\
\left(\frac{0,877}{\lambda_{0}^{2}}\right) & \text { Para } \lambda_{0}>1,5\end{cases} \\
& \lambda_{0}=\sqrt{\frac{A \cdot f_{y}}{N_{e}}}
\end{aligned}
$$

A é a área bruta da seção transversal da barra e $N_{e}$ é a força normal de flambagem global elástica da barra para perfil com seção monossimétrica que é o menor valor entre $N_{e 2}$ (força normal de flambagem por flexão em relação ao eixo 2 - Figura 1) e $N_{e 1 z}$ (força normal de flambagem por flexo-torção).

A força normal de flambagem por flexão em relação ao eixo $2\left(N_{e 2}\right)$ é calculada pela Equação (4).

$$
N_{e 2}=\frac{\pi^{2} E I_{2}}{\left(K_{2} L\right)^{2}}
$$

$I_{2}$ é o momento de inércia em relação ao eixo 2 e $K_{2} L$ é o comprimento efetivo de flambagem por flexão em relação ao eixo 2 (Figura 1).

A força normal de flambagem por flexo-torção de barra com seção transversal monossimétrica carregada pelo centroide $\left(N_{e 1 z}\right)$ é determinada de acordo com a Teoria 
da Estabilidade Elástica (Timoshenko e Gere, 1961 e Chajes e Winter, 1965), calculada pelas Equações (5), (6) e (7).

$$
\begin{aligned}
& N_{e 1 z}=\frac{N_{e l}+N_{e z}}{2\left[1-\left(x_{0} / r_{0}\right)^{2}\right]}\left[1-\sqrt{1-\frac{4 \mathrm{~N}_{e l} N_{e z}\left[1-\left(x_{0} / r_{0}\right)^{2}\right]}{\left(N_{e l}+N_{e z}\right)^{2}}}\right] \\
& N_{e l}=\frac{\pi^{2} E I_{1}}{\left(K_{1} L\right)^{2}} \\
& N_{e z}=\frac{1}{r_{0}^{2}}\left[\frac{\pi^{2} E C_{w}}{\left(K_{z} L\right)^{2}}+G J\right]
\end{aligned}
$$

$N_{e 1}$ é a força normal de flambagem elástica por flexão em relação ao eixo 1 (Figura 1), $N_{e z}$ é a força normal de flambagem elástica por torção, $x_{0}$ é a distância do centro de gravidade ao centro de torção, $r_{0}$ é o raio polar de giração, $l_{1}$ é o momento de inércia em relação ao eixo $1, K_{1} L$ é o comprimento efetivo de flambagem por flexão em relação ao eixo $1, K_{z} L$ é o comprimento efetivo de flambagem por torção, $J$ é o momento de inércia à torção uniforme, $C_{w}$ é a constante de empenamento da seção e G é o módulo de elasticidade transversal.

A área efetiva da seção transversal $\left(A_{e f}\right)$ é calculada com base no Método da Largura Efetiva (MLE) neste trabalho, utilizando a expressão de Winter et al. (1947) na forma parametrizada, de acordo com as Equações (8) e (9).

$$
\begin{aligned}
& b_{e f}=b\left(1-0,22 / \lambda_{p}\right) / \lambda_{p} \\
& \lambda_{p}=\frac{b / t}{0,95(k E / \sigma)^{0,5}}
\end{aligned}
$$

$b_{e f}$ é a largura efetiva do elemento, $b$ é a largura do elemento, $t$ é a espessura do elemento, $\lambda_{p}$ é o índice de esbeltez reduzido do elemento, $E$ é o módulo de elasticidade do aço, $k$ é o coeficiente de flambagem local do elemento e $\sigma$ a tensão normal.

\section{Resultados de Análise Experimental}

Os resultados de ensaios experimentais utilizados no trabalho foram obtidos por Young (2005). Foram ensaiadas três seções de cantoneiras enrijecidas submetidas à compressão centrada. As cantoneiras foram fabricadas com chapas de aço de alta resistência com revestimento de zinco. Nas extremidades das barras foram soldadas 
chapas de aço com espessura de $25 \mathrm{~mm}$ para assegurar o pleno contato entre o perfil e o dispositivo de aplicação de força. As barras foram ensaiadas com extremidades engastadas (sem rotação). Os resultados dos ensaios foram comparados com os procedimentos normativos da North American Specification (NAS:2001) e com a Australian/New Zealand Standard (AS/NZS: 1996). Os procedimentos apresentados pelas duas normas são idênticos ao procedimento da ABNT NBR 14762:2010, para compressão centrada. No entanto, as duas normas recomendam que cotoneiras com abas esbeltas sejam dimensionadas considerando um momento adicional igual ao carregamento centrado multiplicado por uma excentricidade de $L_{b a r r a} / 1000$, sendo $L_{\text {barra }}$ o comprimento da barra. Ou seja, as barras devem ser dimensionadas para flexocompressão. As propriedades geométricas e mecânicas das seções ensaiadas estão na Tabela 1.

Tabela 1 - Propriedades geométricas e mecânicas das seções ensaiadas

\begin{tabular}{|c|c|c|c|c|c|c|c|}
\hline Seção & $b_{f}(m m)$ & $D(\mathrm{~mm})$ & $t(\mathrm{~mm})$ & $r_{i}(\mathrm{~mm})$ & $\mathrm{f}_{\mathrm{y}}(\mathrm{MPa})$ & $\mathrm{f}_{\mathrm{u}}(\mathrm{MPa})$ & $\mathrm{E}(\mathrm{MPa})$ \\
\hline Le $70 \times 17 \times 1,20$ & 72,3 & 17,0 & 1,20 & 2,60 & 580 & 595 & 211000 \\
\hline Le $70 \times 17 \times 1,50$ & 73,5 & 16,8 & 1,49 & 2,60 & 505 & 550 & 212000 \\
\hline Le $70 \times 17 \times 1,90$ & 73,6 & 17,7 & 1,89 & 2,60 & 495 & 535 & 213000 \\
\hline
\end{tabular}

$b_{f}$ é a largura da aba. $D$ é a largura do enrijecedor. t é a espessura da aba e do enrijecedor. $r_{i}$ é o raio interno de dobramento. $\mathrm{f}_{\mathrm{y}}$ é a resistência ao escoamento do aço. $\mathrm{f}_{\mathrm{u}}$ é a resistência à ruptura do aço.

Os resultados dos ensaios experimentais e a comparação com os obtidos pelas normas (NAS:2001 e AS/NZS: 1996) são apresentados nas Tabelas 2, 3 e 4. Vale ressaltar que os valores foram calculados considerando o momento adicional recomendado.

Os resultados experimentais mostram que a NAS:2001 e a AS/NZS4600:1996, para cálculo de cantoneiras enrijecidas de aço formadas a frio, são conservadoras, com exceção das barras mais curtas de espessura de 1,20 mm. A flexo-torção é prevista pelas normas, o que está de acordo com os resultados experimentais, com exceção de comprimentos curtos, em que este modo de falha não foi observado. A flexão é observada em todas as barras com comprimento igual ou superior a $2500 \mathrm{~mm}$, mas as normas não preveem este modo de falha. De acordo com Young (2005) a excentricidade adicional recomendada pelas normas deve ser ignorada no dimensionamento de barras de cantoneiras enrijecidas. Os resultados das normas sem a consideração do momento adicional são apresentados nas Tabelas 5, 6 e 7 onde são comparados com os resultados experimentais e numéricos. 
Tabela 2 - Resultados dos ensaios realizados por Young (2005): seção Le 70x17x1,20

\begin{tabular}{ccccccc}
\hline $\begin{array}{c}\text { No } \\
\text { ensaio }\end{array}$ & $\begin{array}{c}\mathbf{L}_{\text {barra }} \\
(\mathbf{m m})\end{array}$ & $\begin{array}{c}\mathbf{N}_{\text {Exp }} \\
(\mathbf{k N})\end{array}$ & $\begin{array}{c}\mathbf{N}_{\text {NAS }} \\
(\mathbf{k N})\end{array}$ & $\mathbf{N}_{\text {Exp }} / \mathbf{N}_{\text {NAS }}$ & \multicolumn{2}{c}{ Modo de falha } \\
\cline { 6 - 7 } & 250 & 59,7 & 60,6 & 0,99 & $\mathrm{~L}$ & $\mathrm{E}+\mathrm{FT}$ \\
2 & 250 & 57,9 & 60,6 & 0,96 & $\mathrm{~L}$ & $\mathrm{~L}+\mathrm{FT}$ \\
3 & 1000 & 40,3 & 32,8 & 1,23 & $\mathrm{~L}+\mathrm{FT}$ & $\mathrm{L}+\mathrm{FT}$ \\
4 & 1500 & 33,4 & 18,9 & 1,77 & $\mathrm{~L}+\mathrm{FT}$ & $\mathrm{FT}$ \\
5 & 2000 & 30,3 & 11,8 & 2,58 & $\mathrm{~L}+\mathrm{FT}$ & $\mathrm{FT}$ \\
6 & 2500 & 27,2 & 8,5 & 3,20 & $\mathrm{~L}+\mathrm{F}+\mathrm{FT}$ & $\mathrm{FT}$ \\
7 & 3000 & 22,3 & 6,7 & 3,33 & $\mathrm{~L}+\mathrm{F}+\mathrm{FT}$ & $\mathrm{FT}$ \\
8 & 3500 & 19,4 & 5,6 & 3,46 & $\mathrm{~L}+\mathrm{F}+\mathrm{FT}$ & $\mathrm{FT}$ \\
\hline
\end{tabular}

Lbarra é o comprimento da barra.

$\mathrm{L}=$ instabilidade local; $\mathrm{F}$ = instabilidade por flexão e $\mathrm{FT}$ = instabilidade por flexo-torção.

$\mathrm{N}_{\text {Exp }}$ é a força resistente obtida nos ensaios experimentais.

$\mathrm{N}_{\text {NAS }}$ é a força resistente calculada de acordo com as normas NAS:2001 e AS/NZS: 1996.

Tabela 3 - Resultados dos ensaios realizados por Young (2005): seção Le 70x17x1,50

\begin{tabular}{ccccccc}
\hline $\begin{array}{c}\text { No } \\
\text { ensaio }\end{array}$ & $\begin{array}{c}\mathbf{L}_{\text {barra }} \\
(\mathbf{m m})\end{array}$ & $\begin{array}{c}\mathbf{N}_{\text {Exp }} \\
(\mathbf{k N})\end{array}$ & $\begin{array}{c}\mathbf{N}_{\text {NAS }} \\
(\mathbf{k N})\end{array}$ & $\mathbf{N}_{\text {Exp }} / \mathbf{N}_{\text {AS }}$ & \multicolumn{2}{c}{ Modo de falha } \\
\cline { 6 - 7 } & 250 & 84,6 & 74,4 & 1,14 & Experimental & Normas \\
\hline 2 & 625 & 74,9 & 60,4 & 1,24 & $\mathrm{FT}$ & $\mathrm{L}+\mathrm{FT}$ \\
3 & 1000 & 54,9 & 41,6 & 1,32 & $\mathrm{FT}$ & $\mathrm{L}+\mathrm{FT}$ \\
4 & 1500 & 48,7 & 24,6 & 1,98 & $\mathrm{FT}$ & $\mathrm{FT}$ \\
5 & 2000 & 41,5 & 16,0 & 2,59 & $\mathrm{FT}$ & $\mathrm{FT}$ \\
6 & 2500 & 39,4 & 12,1 & 3,26 & $\mathrm{~F}+\mathrm{FT}$ & $\mathrm{FT}$ \\
7 & 3000 & 37,3 & 9,9 & 3,77 & $\mathrm{~F}+\mathrm{FT}$ & $\mathrm{FT}$ \\
8 & 3500 & 30,9 & 8,6 & 3,59 & $\mathrm{~F}+\mathrm{FT}$ & $\mathrm{FT}$ \\
\hline
\end{tabular}

Lbarra é o comprimento da barra.

$\mathrm{L}=$ instabilidade local; $\mathrm{F}$ = instabilidade por flexão e FT = instabilidade por flexo-torção.

$\mathrm{N}_{\mathrm{Exp}}$ é a força resistente obtida nos ensaios experimentais.

N NAS é a força resistente calculada de acordo com as normas NAS:2001 e AS/NZS: 1996.

Tabela 4 - Resultados dos ensaios realizados por Young (2005): seção Le 70x17×1,90

\begin{tabular}{ccccccc}
\hline $\begin{array}{c}\text { No } \\
\text { ensaio }\end{array}$ & $\begin{array}{c}\mathbf{L}_{\text {barra }} \\
(\mathbf{m m})\end{array}$ & $\begin{array}{c}\mathbf{N}_{\text {Exp }} \\
(\mathbf{k N})\end{array}$ & $\begin{array}{c}\mathbf{N}_{\text {NAs }} \\
(\mathbf{k N})\end{array}$ & $\mathbf{N}_{\text {Exp }} / \mathbf{N}_{\text {AS }}$ & \multicolumn{2}{c}{ Modo de falha } \\
\cline { 6 - 7 } & 250 & 130,9 & 103,1 & 1,27 & Experimental & Normas \\
\hline 2 & 625 & 103,5 & 85,2 & 1,21 & $\mathrm{FT}$ & $\mathrm{L}+\mathrm{FT}$ \\
3 & 1000 & 79,8 & 65,1 & 1,23 & $\mathrm{FT}$ & $\mathrm{L}+\mathrm{FT}$ \\
4 & 1000 & 79,8 & 65,1 & 1,23 & $\mathrm{FT}$ & $\mathrm{FT}$ \\
5 & 1500 & 69,3 & 37,5 & 1,85 & $\mathrm{FT}$ & $\mathrm{FT}$ \\
6 & 2000 & 64,3 & 25,5 & 2,52 & $\mathrm{FT}$ & $\mathrm{FT}$ \\
7 & 2500 & 52,9 & 19,9 & 2,66 & $\mathrm{~F}+\mathrm{FT}$ & $\mathrm{FT}$ \\
8 & 3000 & 47,5 & 16,9 & 2,81 & $\mathrm{~F}+\mathrm{FT}$ & $\mathrm{FT}$ \\
9 & 3500 & 40,4 & 15,0 & 2,69 & $\mathrm{~F}+\mathrm{FT}$ & $\mathrm{FT}$ \\
\hline
\end{tabular}

Lbarra é o comprimento da barra.

$\mathrm{L}=$ instabilidade local; $\mathrm{F}$ = instabilidade por flexão e FT = instabilidade por flexo-torção.

$\mathrm{N}_{\mathrm{Exp}}$ é a força resistente obtida nos ensaios experimentais.

$\mathrm{N}_{\text {NAS }}$ é a força resistente calculada de acordo com as normas NAS:2001 e AS/NZS: 1996. 


\section{Análise Numérica}

As simulações numéricas foram realizadas utilizando um programa baseado no Método dos Elementos Finitos - ANSYS v13.0. As simulações foram realizadas utilizando o elemento SHELL 181 para a modelagem das cantoneiras enrijecidas, chapas de extremidade e "perfis T" das extremidades. De acordo com os tópicos de ajuda do ANSYS, o elemento é ideal para análise não-linear de cascas de pequena espessura sujeitas a grandes deformações e rotações.

Para a resolução do sistema não-linear foi utilizado o método iterativo e incremental Newton-Raphson Completo que atualiza a matriz de rigidez tangente a cada iteração. O carregamento foi incrementado utilizando-se a ferramenta do ANSYS conhecida como "Automatic Load Stepping". Esta ferramenta atualiza de forma automática o incremento a ser acrescido, introduzindo limites superior e inferior de incrementos.

Foi utilizado o critério de convergência em termo dos deslocamentos para verificar se a solução obtida possui a precisão julgada suficiente. Segundo Lourenço (1999), o critério de convergência em termos de deslocamentos é dado por $\|\delta u\|<\beta\|u\|$ em que $\delta u$ é a correção iterativa do deslocamento, $u$ é o deslocamento total e $\beta$ é a tolerância ou erro máximo admitido. No presente trabalho, o valor de tolerância $(\beta)$ adotado foi de 0,001 (0,1\%), que é o default do ANSYS. Para melhor convergência dos modelos foi adotada a ferramenta "Line-Search", que faz a estimativa de uma solução exterior ao raio de convergência do método de Newton-Raphson. Com relação ao modelo reológico do material, foi utilizado o modelo trilinear, já que este apresenta resultados bastante satisfatórios, conforme apresentado por Maia (2008).

Todos os elementos foram adotados como sendo aproximadamente quadrados, com lado de aproximadamente $1 \mathrm{~cm}$, com exceção dos cantos que foram discretizados em dois elementos. Todas as seções foram construídas utilizando as dimensões da linha de esqueleto e cantos arredondados. Assim como na análise experimental, foram modeladas chapas de $25 \mathrm{~mm}$ nas extremidades das barras submetidas à compressão centrada. As duas extremidades foram engastadas, com todos os graus de liberdade dos nós sendo restringidos, permitindo apenas o deslocamento longitudinal (Uz) em uma das extremidades, onde foi aplicado o carregamento. Vale ressaltar que todos os nós dessa extremidade tiveram o deslocamento acoplado nessa mesma direção (Uz). 0 
carregamento foi aplicado no nó de menor numeração dessa extremidade. A Figura 2 ilustra como foram modeladas as barras submetidas à compressão centrada no ANSYS.

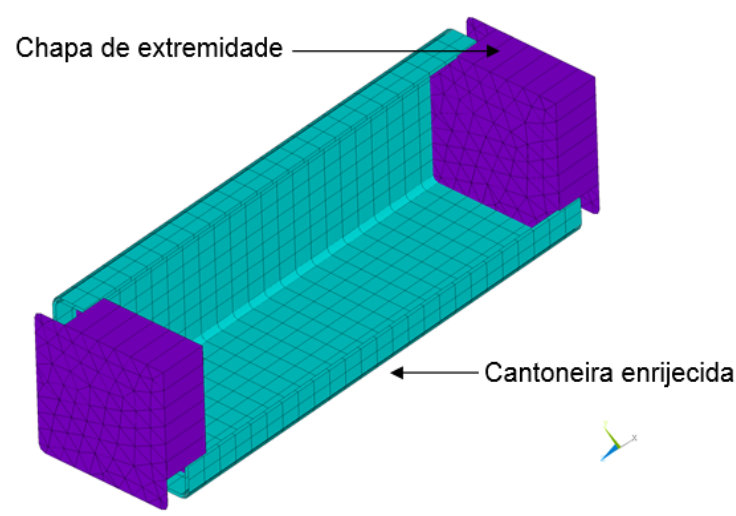

(a)

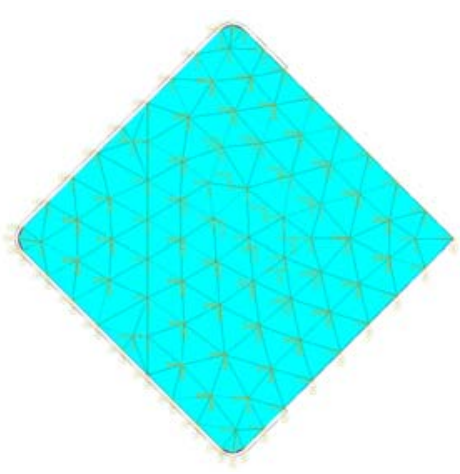

(b)

Figura 2 - Cantoneira enrijecida e ilustração da malha para a seção Le 70x17×1,90 (Compressão centrada) (a) Vista geral (b) Detalhe da chapa de extremidade com as restrições

Para os modelos conectados pela aba, os "perfis $T$ " das extremidades foram construídos de forma a coincidir suas malhas com a malha da cantoneira, no entanto, foram construídos com um espaço de $0,1 \mathrm{~mm}$ da cantoneira, de forma que não houvesse sobreposição de nós. Posteriormente, foram realizados acoplamentos de deslocamentos dos nós coincidentes que delimitam a cantoneira e os "perfis T" nas três direções, simulando duas linhas de solda, sendo estes nós responsáveis pela transmissão do carregamento dos "perfis T" para a cantoneira, como mostra a Figura 3.

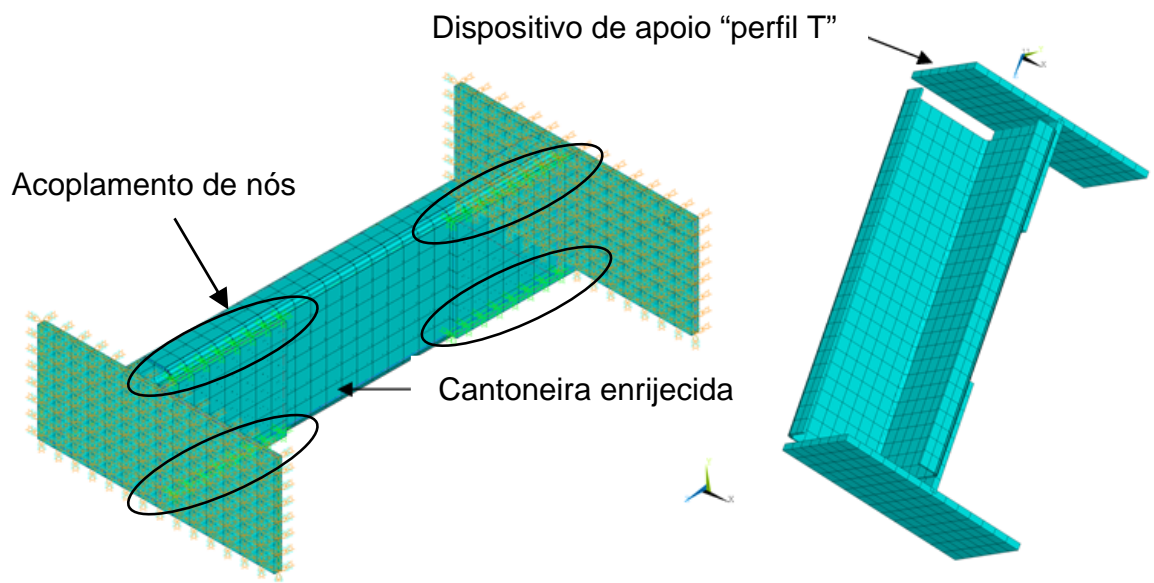

Figura 3 - Ilustração da malha para a seção Le 70x17x1,90 (Compressão excêntrica)

As condições nas extremidades dos "perfis T" (mesas) foram as mesmas adotadas para as barras com extremidades engastadas (sem rotação). Assim, as rotações da 
cantoneira são provenientes apenas da ligação do perfil com os dispositivos de apoio das extremidades (perfis T). Nesse caso, o carregamento foi aplicado no nó de menor numeração da mesa do "perfil T" da extremidade.

O modo como as imperfeições geométricas iniciais foram adotadas segue o mesmo princípio utilizado por Chodraui (2006), Almeida (2007) e Maia (2008). Inicialmente, foi realizada uma análise de autovalor utilizando o software ANSYS, que fornece como resposta um autovalor (valor de força crítica) e um autovetor (deformada da barra). Com isso, buscou-se identificar os modos críticos isolados: local, globais de flexotorção e de flexão para cantoneira enrijecida. A partir da configuração deformada referente aos modos críticos escolhidos para cada caso, foi adotado um critério a fim de se aumentar ou reduzir essa amplitude, obtendo-se uma nova geometria de todos os nós da malha de elementos finitos da barra.

Com relação à amplitude das imperfeições geométricas iniciais foram utilizados os valores de imperfeições apresentados por Schafer \& Peköz (1998). Optou-se, sempre, pela combinação mais desfavorável na sobreposição de imperfeições. Foram adotadas imperfeições do tipo $1\left(d_{1}\right)$ associadas ao modo local, do tipo $2\left(d_{2}\right)$ associadas ao modo de flexo-torção, conforme apresentado por Schafer \& Peköz (1998), e Lbarra/1500 associada ao modo de flexão.

\section{Resultados}

\subsection{Cantoneira enrijecida submetida a compressão centrada}

Nas Tabelas 5, 6 e 7 são apresentados os resultados da análise numérica das seções submetidas a compressão centrada comparados com os resultados experimentais obtidos por Young (2005) e com as previsões teóricas calculadas de acordo com a ABNT NBR 14762:2010. Vale ressaltar que o procedimento da ABNT NBR 14762:2010 é idêntico aos procedimentos da NAS:2001 e AS/NZS: 1996, sem a adição do momento recomendado para cantoneiras com abas esbeltas. As simulações foram realizadas com a inserção de imperfeições de $0,14 t\left(d_{1}\right)$ associada ao modo local, $0,64 t\left(d_{2}\right)$ para o modo de flexo-torção e Lbarra/1500 para o modo de flexão (Imperfeições 1). Foram também realizadas simulações sem a inserção de imperfeições geométricas iniciais de flexo-torção e flexão para barras com comprimento igual a $250 \mathrm{~mm}$ e sem a inserção 
de imperfeições geométricas inicias local para barras com comprimento maior que 250 mm (Imperfeições 2).

Tabela 5 - Comparação dos resultados da análise numérica com os obtidos experimentalmente por Young (2005) e da ABNT NBR 14762:2010: seção Le 70×17×1,20

\begin{tabular}{|c|c|c|c|c|c|c|c|c|c|}
\hline $\begin{array}{l}\mathrm{L}_{\text {barra }} \\
(\mathrm{mm})\end{array}$ & $\begin{array}{l}N_{\text {Exp }} \\
(k N)\end{array}$ & $\begin{array}{l}\text { Modo } \\
\text { de Falha }\end{array}$ & $\begin{array}{l}N_{\mathrm{EF}} \\
(\mathrm{kN})\end{array}$ & $\begin{array}{l}\text { Modo } \\
\text { de Falha }\end{array}$ & $\mathbf{N}_{\mathrm{Exp}} / \mathrm{N}_{\mathrm{EF}}$ & $\begin{array}{l}N_{c, R}{ }^{1} \\
(k N)\end{array}$ & $N_{E F} / N_{C, R}{ }^{1}$ & $\begin{array}{l}N_{c, R}{ }^{2} \\
(k N)\end{array}$ & $N_{E F} / N_{C, R}{ }^{2}$ \\
\hline
\end{tabular}

Extremidades engastadas $\left(\mathrm{K}_{1}=\mathrm{K}_{2}=\mathrm{K}_{\mathrm{z}}=0,5\right)$

$d_{1}=0,14 t, d_{2}=0,64 t$ e Lbarra $/ 1500$ para todas as barras (Imperfeições 1 )

\begin{tabular}{cccccccccc}
\hline 250 & 59,7 & L & 57,8 & L & 1,03 & 71,2 & 0,81 & 74,7 & 0,77 \\
250 & 57,9 & L & 57,8 & L & 1,00 & 71,2 & 0,81 & 74,7 & 0,77 \\
1000 & 40,3 & L+FT & 40,2 & L+FT & 1,00 & 33,8 & 1,19 & 70,1 & 0,57 \\
1500 & 33,4 & L+FT & 34,1 & L+F+FT & 0,98 & 19,2 & 1,78 & 64,3 & 0,53 \\
2000 & 30,3 & L+FT & 31,2 & L+F+FT & 0,97 & 12,0 & 2,60 & 57,0 & 0,55 \\
2500 & 27,2 & L+F+FT & 28,8 & L+F+FT & 0,94 & 8,6 & 3,35 & 48,9 & 0,59 \\
3000 & 22,3 & L+F+FT & 26,4 & L+F+FT & 0,84 & 6,8 & 3,88 & 40,5 & 0,65 \\
3500 & 19,4 & L+F+FT & 23,1 & L+F+FT & 0,84 & 5,7 & 4,05 & 32,5 & 0,71 \\
\hline & & & & Média & $\mathbf{0 , 9 5}$ & & $\mathbf{2 , 3 1}$ & & $\mathbf{0 , 6 4}$ \\
& & & & CV & $\mathbf{0 , 0 8}$ & & $\mathbf{1 , 3 5}$ & & $\mathbf{0 , 1 0}$ \\
\hline
\end{tabular}

Extremidades engastadas $\left(K_{1}=K_{2}=K_{z}=0,5\right)$

$\mathrm{d}_{1}=0,14 \mathrm{t}$ para barra de $250 \mathrm{~mm}$

$\mathrm{d}_{2}=0,64 \mathrm{t}$ e $\mathrm{L}_{\text {barra }} / 1500$ para barras acima de $250 \mathrm{~mm}$ (Imperfeições 2 )

\begin{tabular}{cccccccccc}
\hline 250 & 59,7 & L & 57,7 & L & 1,03 & 71,2 & 0,81 & 74,7 & 0,77 \\
250 & 57,9 & L & 57,7 & L & 1,00 & 71,2 & 0,81 & 74,7 & 0,77 \\
1000 & 40,3 & L+FT & 42,1 & L+FT & 0,96 & 33,8 & 1,25 & 70,1 & 0,60 \\
1500 & 33,4 & L+FT & 35,1 & L+F+FT & 0,95 & 19,2 & 1,83 & 64,3 & 0,55 \\
2000 & 30,3 & L+FT & 32,0 & L+F+FT & 0,95 & 12,0 & 2,67 & 57,0 & 0,56 \\
2500 & 27,2 & L+F+FT & 30,0 & L+F+FT & 0,91 & 8,6 & 3,49 & 48,9 & 0,61 \\
3000 & 22,3 & L+F+FT & 27,4 & F+FT & 0,81 & 6,8 & 4,03 & 40,5 & 0,68 \\
3500 & 19,4 & L+F+FT & 24,6 & F+FT & 0,79 & 5,7 & 4,32 & 32,5 & 0,76 \\
\hline & & & & Média & $\mathbf{0 , 9 3}$ & & $\mathbf{2 , 4 0}$ & & $\mathbf{0 , 6 6}$ \\
& & & & CV & $\mathbf{0 , 0 9}$ & & $\mathbf{1 , 4 3}$ & & $\mathbf{0 , 1 0}$ \\
\hline
\end{tabular}

Lbarra é o comprimento da barra.

$N_{\text {Exp }}$ é a força normal centrada obtida experimentalmente por Young (2005).

$\mathrm{N}_{\mathrm{EF}}$ é a força normal centrada obtida por elementos finitos.

$\mathrm{L}=$ instabilidade local; $\mathrm{F}$ = instabilidade por flexão e FT = instabilidade por flexo-torção.

CV = coeficiente de variação.

$\mathrm{N}_{\mathrm{c}, \mathrm{R}}{ }^{1}$ é calculado de acordo com a ABNT NBR 14762:2010 utilizando o mínimo entre flexão e flexotorção para o modo de instabilidade global (Procedimento recomendado).

$\mathrm{N}_{c, R^{2}}$ é calculado de acordo com a ABNT NBR 14762:2010 utilizando apenas o modo global de flexão. 
Tabela 6 - Comparação dos resultados da análise numérica com os obtidos experimentalmente por Young (2005) e da ABNT NBR 14762:2010: seção Le 70x17x1,50

\begin{tabular}{|c|c|c|c|c|c|c|c|c|c|}
\hline $\begin{array}{l}\text { L } \text { Larra } \\
(\mathrm{mm})\end{array}$ & $\begin{array}{l}N_{\text {Exp }} \\
(k N)\end{array}$ & $\begin{array}{l}\text { Modo } \\
\text { de Falha }\end{array}$ & $\begin{array}{l}\mathrm{N}_{\mathrm{EF}} \\
(\mathrm{kN})\end{array}$ & $\begin{array}{l}\text { Modo de } \\
\text { Falha }\end{array}$ & $\mathbf{N}_{\mathrm{Exp}} / \mathbf{N}_{\mathrm{EF}}$ & $\begin{array}{l}N_{c, R}{ }^{1} \\
(k N)\end{array}$ & $N_{E F} / N_{c, R}{ }^{1}$ & $\begin{array}{l}N_{c, R}{ }^{2} \\
(\mathbf{k N})\end{array}$ & $\mathbf{N}_{\mathrm{EF}} / \mathbf{N}_{\mathrm{C}, \mathrm{R}}{ }^{2}$ \\
\hline \multicolumn{10}{|c|}{ Extremidades engastadas $\left(\mathrm{K}_{1}=\mathrm{K}_{2}=\mathrm{K}_{2}=0,5\right)$} \\
\hline \multicolumn{10}{|c|}{$\mathrm{d}_{1}=0,14 \mathrm{t}, \mathrm{d}_{2}=0,64 \mathrm{t}$ e $\mathrm{L}_{\text {barra/ }} / 1500$ para todas as barras (Imperfeições 1 ) } \\
\hline 250 & 84,6 & L & 72,4 & L & 1,17 & 87,5 & 0,83 & 91,5 & 0,79 \\
\hline 625 & 74,9 & FT & 67,8 & $\mathrm{~L}+\mathrm{FT}$ & 1,10 & 68,7 & 0,99 & 89,7 & 0,76 \\
\hline 1000 & 54,9 & FT & 52,1 & $\mathrm{~L}+\mathrm{FT}$ & 1,05 & 45,4 & 1,15 & 86,6 & 0,60 \\
\hline 1500 & 48,7 & $\mathrm{FT}$ & 45,2 & $\mathrm{~L}+\mathrm{F}+\mathrm{FT}$ & 1,08 & 25,2 & 1,79 & 80,4 & 0,56 \\
\hline 2000 & 41,5 & FT & 41,8 & $\mathrm{~L}+\mathrm{F}+\mathrm{FT}$ & 0,99 & 16,4 & 2,55 & 72,6 & 0,58 \\
\hline 2500 & 39,4 & $\mathrm{~F}+\mathrm{FT}$ & 38,6 & $\mathrm{~L}+\mathrm{F}+\mathrm{FT}$ & 1,02 & 12,3 & 3,14 & 63,6 & 0,61 \\
\hline 3000 & 37,3 & $\mathrm{~F}+\mathrm{FT}$ & 34,7 & $\mathrm{~L}+\mathrm{F}+\mathrm{FT}$ & 1,08 & 10,1 & 3,44 & 54,0 & 0,64 \\
\hline \multirow[t]{3}{*}{3500} & 30,9 & $\mathrm{~F}+\mathrm{FT}$ & 30,1 & $\mathrm{~L}+\mathrm{F}+\mathrm{FT}$ & 1,03 & 8,7 & 3,46 & 44,3 & 0,68 \\
\hline & & & & Média & 1,06 & & 2,17 & & 0,65 \\
\hline & & & & CV & 0,05 & & 1,12 & & 0,08 \\
\hline
\end{tabular}

Extremidades engastadas $\left(\mathrm{K}_{1}=\mathrm{K}_{2}=\mathrm{K}_{2}=0,5\right)$

$\mathrm{d}_{1}=0,14 \mathrm{t}$ para barra de $250 \mathrm{~mm}$

$\mathrm{d}_{2}=0,64 \mathrm{t}$ e $\mathrm{L}_{\text {barra }} / 1500$ para barras acima de $250 \mathrm{~mm}$ (Imperfeições 2)

\begin{tabular}{cccccccccc}
\hline 250 & 84,6 & L & 79,4 & L & 1,06 & 87,5 & 0,91 & 91,5 & 0,87 \\
625 & 74,9 & FT & 70,1 & FT & 1,07 & 68,7 & 1,02 & 89,7 & 0,78 \\
1000 & 54,9 & FT & 54,3 & FT & 1,01 & 45,4 & 1,20 & 86,6 & 0,63 \\
1500 & 48,7 & FT & 48,2 & F+FT & 1,01 & 25,2 & 1,91 & 80,4 & 0,60 \\
2000 & 41,5 & FT & 44,8 & F+FT & 0,93 & 16,4 & 2,73 & 72,6 & 0,62 \\
2500 & 39,4 & F+FT & 40,9 & F+FT & 0,96 & 12,3 & 3,33 & 63,6 & 0,64 \\
3000 & 37,3 & F+FT & 36,0 & F+FT & 1,04 & 10,1 & 3,56 & 54,0 & 0,67 \\
3500 & 30,9 & F+FT & 30,9 & F+FT & 1,00 & 8,7 & 3,55 & 44,3 & 0,70 \\
\hline & & & & Média & $\mathbf{1 , 0 1}$ & & $\mathbf{2 , 2 8}$ & & $\mathbf{0 , 6 9}$ \\
& & & & CV & $\mathbf{0 , 0 5}$ & & $\mathbf{1 , 1 6}$ & & $\mathbf{0 , 0 9}$ \\
\hline
\end{tabular}

Lbarra é o comprimento da barra.

$N_{\text {Exp }}$ é a força normal centrada obtida experimentalmente por Young (2005).

$\mathrm{N}_{\mathrm{EF}}$ é a força normal centrada obtida por elementos finitos.

$\mathrm{L}=$ instabilidade local; $\mathrm{F}$ = instabilidade por flexão e FT = instabilidade por flexo-torção.

CV = coeficiente de variação.

$\mathrm{N}_{\mathrm{c}, \mathrm{R}}{ }^{1}$ é calculado de acordo com a ABNT NBR 14762:2010 utilizando o mínimo entre flexão e flexotorção para o modo de instabilidade global (Procedimento recomendado).

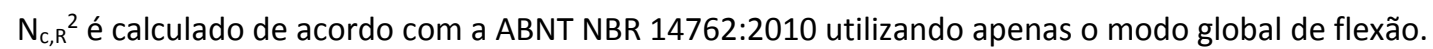


Tabela 7 - Comparação dos resultados da análise numérica com os obtidos experimentalmente por Young (2005) e da ABNT NBR 14762:2010: seção Le 70x17x1,90

\begin{tabular}{|c|c|c|c|c|c|c|c|c|c|}
\hline $\begin{array}{l}\text { Lbarra } \\
(\mathrm{mm})\end{array}$ & $\begin{array}{l}N_{\text {Exp }} \\
\text { (kN) }\end{array}$ & $\begin{array}{l}\text { Modo } \\
\text { de } \\
\text { Falha }\end{array}$ & $\begin{array}{l}N_{\mathrm{EF}} \\
(\mathrm{kN})\end{array}$ & $\begin{array}{l}\text { Modo } \\
\text { de Falha }\end{array}$ & $\mathrm{N}_{\mathrm{Exp}} / \mathrm{N}_{\mathrm{EF}}$ & $\begin{array}{l}N_{c, R}{ }^{1} \\
\text { (kN) }\end{array}$ & $\mathbf{N}_{\mathrm{EF}} / \mathbf{N}_{\mathrm{C}, \mathrm{R}}{ }^{1}$ & $\begin{array}{l}N_{c, R}{ }^{2} \\
(k N)\end{array}$ & $\mathbf{N}_{\mathrm{EF}} / \mathbf{N}_{\mathrm{C}, \mathrm{R}}{ }^{2}$ \\
\hline \multicolumn{10}{|c|}{ Extremidades engastadas $\left(K_{1}=K_{2}=K_{2}=0,5\right)$} \\
\hline \multicolumn{10}{|c|}{$\mathrm{d}_{1}=0,14 \mathrm{t}, \mathrm{d}_{2}=0,64 \mathrm{t}$ e $\mathrm{L}_{\text {barra }} / 1500$ para todas as barras (Imperfeições 1 ) } \\
\hline 250 & 130,9 & $\mathrm{~L}$ & 101,2 & L & 1,29 & 121,9 & 0,83 & 126,8 & 0,80 \\
\hline 625 & 103,5 & FT & 95,4 & $\mathrm{~L}+\mathrm{FT}$ & 1,08 & 98,5 & 0,97 & 124,5 & 0,77 \\
\hline 1000 & 79,8 & FT & 72,8 & $\mathrm{~L}+\mathrm{FT}$ & 1,10 & 69,2 & 1,05 & 120,2 & 0,61 \\
\hline 1500 & 69,3 & FT & 63,2 & $\mathrm{~L}+\mathrm{F}+\mathrm{FT}$ & 1,10 & 38,4 & 1,65 & 111,8 & 0,57 \\
\hline 2000 & 64,3 & FT & 58,1 & $\mathrm{~L}+\mathrm{F}+\mathrm{FT}$ & 1,11 & 26,1 & 2,23 & 101,1 & 0,57 \\
\hline 2500 & 52,9 & $\mathrm{~F}+\mathrm{FT}$ & 52,5 & $\mathrm{~L}+\mathrm{F}+\mathrm{FT}$ & 1,01 & 20,3 & 2,59 & 88,6 & 0,69 \\
\hline 3000 & 47,5 & $\mathrm{~F}+\mathrm{FT}$ & 46,1 & $\mathrm{~L}+\mathrm{F}+\mathrm{FT}$ & 1,03 & 17,1 & 2,70 & 75,3 & 0,61 \\
\hline \multirow[t]{3}{*}{3500} & 40,4 & $\mathrm{~F}+\mathrm{FT}$ & 39,8 & $\mathrm{~L}+\mathrm{F}+\mathrm{FT}$ & 1,01 & 15,2 & 2,62 & 61,8 & 0,64 \\
\hline & & & & Média & 1,09 & & 1,83 & & 0,64 \\
\hline & & & & CV & 0,08 & & 0,80 & & 0,09 \\
\hline
\end{tabular}

Extremidades engastadas $\left(\mathrm{K}_{1}=\mathrm{K}_{2}=\mathrm{K}_{\mathrm{z}}=0,5\right)$

$\mathrm{d}_{1}=0,14 \mathrm{t}$ para barra de $250 \mathrm{~mm}$

$\mathrm{d}_{2}=0,64 \mathrm{t}$ e $\mathrm{L}_{\text {barra }} / 1500$ para barras acima de $250 \mathrm{~mm}$ (Imperfeições 2 )

\begin{tabular}{cccccccccc}
\hline 250 & 130,9 & L & 116,2 & L & 1,13 & 121,9 & 0,95 & 126,8 & 0,92 \\
625 & 103,5 & FT & 97,5 & FT & 1,06 & 98,5 & 0,99 & 124,5 & 0,78 \\
1000 & 79,8 & FT & 74,1 & FT & 1,08 & 69,2 & 1,07 & 120,2 & 0,62 \\
1500 & 69,3 & FT & 66,6 & F+FT & 1,04 & 38,4 & 1,73 & 111,8 & 0,60 \\
2000 & 64,3 & FT & 60,5 & F+FT & 1,06 & 26,1 & 2,32 & 101,1 & 0,60 \\
2500 & 52,9 & F+FT & 53,4 & F+FT & 0,99 & 20,3 & 2,63 & 88,6 & 0,60 \\
3000 & 47,5 & F+FT & 46,5 & F+FT & 1,02 & 17,1 & 2,72 & 75,3 & 0,62 \\
3500 & 40,4 & F+FT & 40,3 & F+FT & 1,00 & 15,2 & 2,65 & 61,8 & 0,65 \\
\hline & & & & Média & $\mathbf{1 , 0 5}$ & & $\mathbf{1 , 8 8}$ & & $\mathbf{0 , 6 7}$ \\
& & & & CV & $\mathbf{0 , 0 4}$ & & $\mathbf{0 , 7 9}$ & & $\mathbf{0 , 1 2}$ \\
\hline
\end{tabular}

Lbarra é o comprimento da barra.

$N_{\text {Exp }}$ é a força normal centrada obtida experimentalmente por Young (2005).

$N_{\mathrm{EF}}$ é a força normal centrada obtida por elementos finitos.

$\mathrm{L}=$ instabilidade local; $\mathrm{F}=$ instabilidade por flexão e FT = instabilidade por flexo-torção.

$\mathrm{CV}=$ coeficiente de variação.

$\mathrm{N}_{c, R}{ }^{1}$ é calculado de acordo com a ABNT NBR 14762:2010 utilizando o mínimo entre flexão e flexotorção para o modo de instabilidade global (Procedimento recomendado).

$\mathrm{N}_{\mathrm{C}, \mathrm{R}}{ }^{2}$ é calculado de acordo com a ABNT NBR 14762:2010 utilizando apenas o modo global de flexão.

Nas Figuras 4, 5 e 6 são apresentados os resultados da análise numérica comparados com os resultados experimentais e com as previsões teóricas calculadas. Nas Figuras 7 
e 8 são apresentados típicos modos de instabilidade observados na análise numérica das barras submetidas à compressão centrada. As mesmas figuras ilustram o panorama de tensões de von Mises observado no instante em que a barra atinge a resistência máxima.

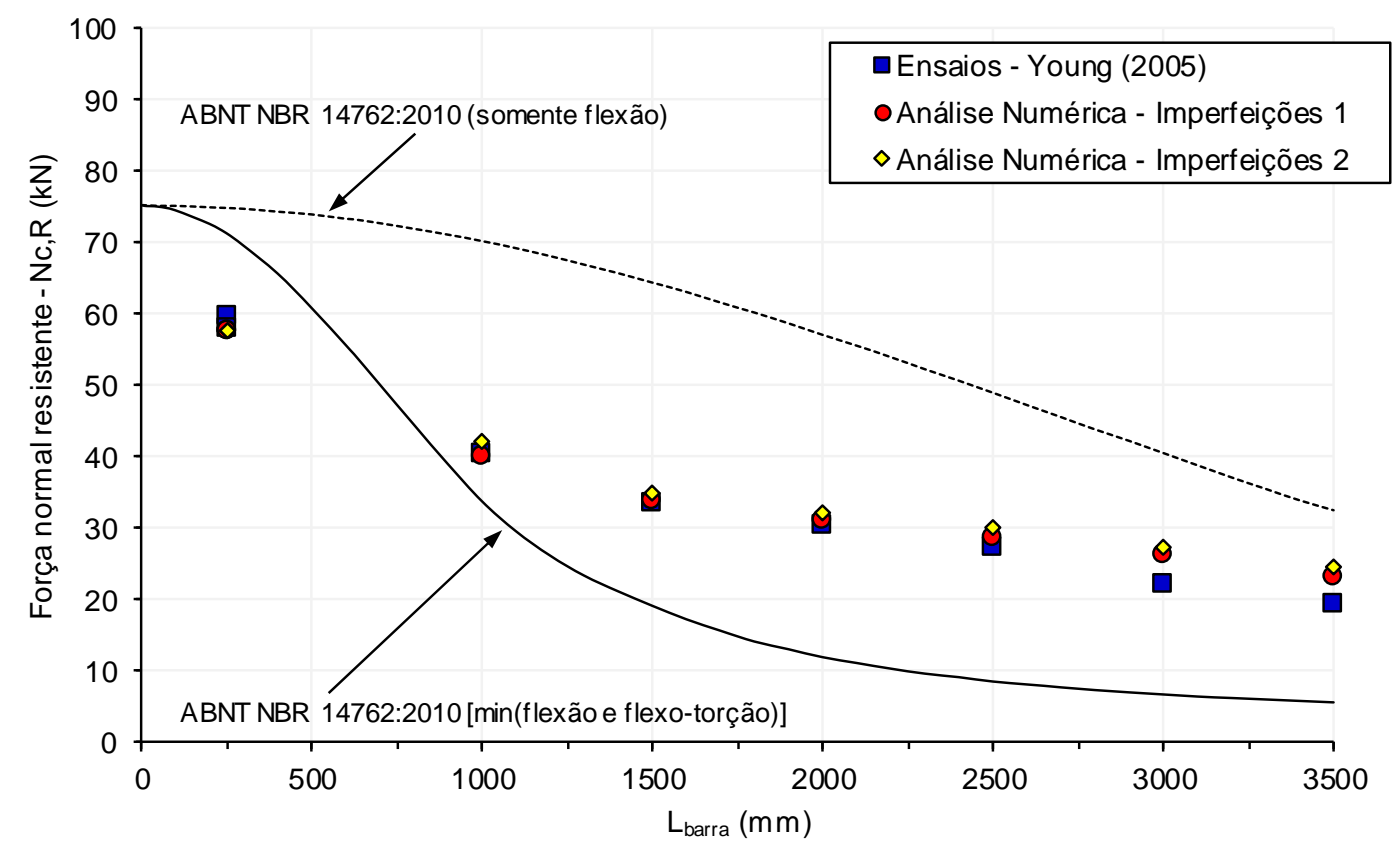

Figura 4 - Comparação dos resultados da análise numérica com os obtidos experimentalmente por Young (2005) e da ABNT NBR 14762:2010: seção Le 70x17×1,20

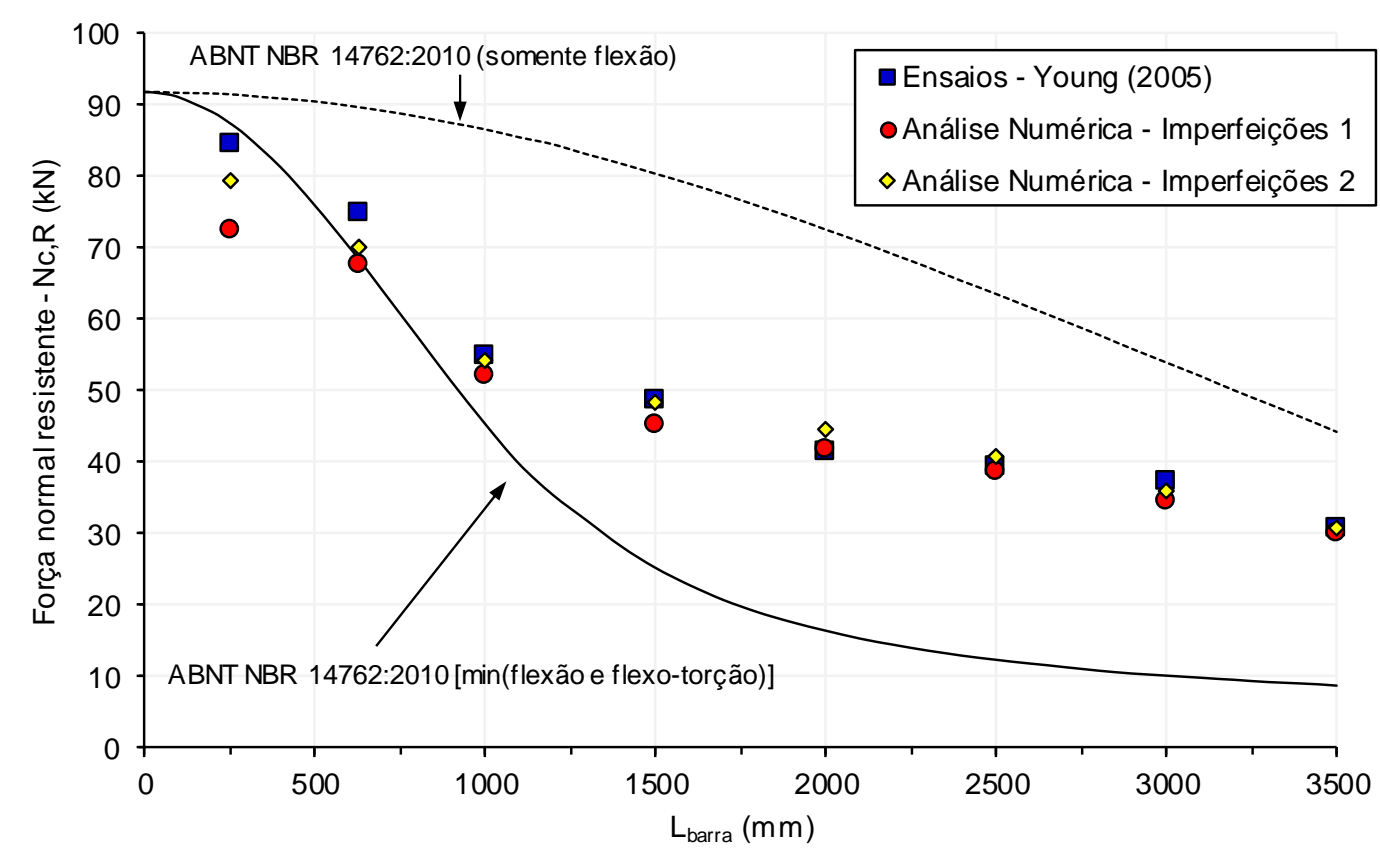

Figura 5 - Comparação dos resultados da análise numérica com os obtidos experimentalmente por Young (2005) e da ABNT NBR 14762:2010: seção Le 70x17x1,50 


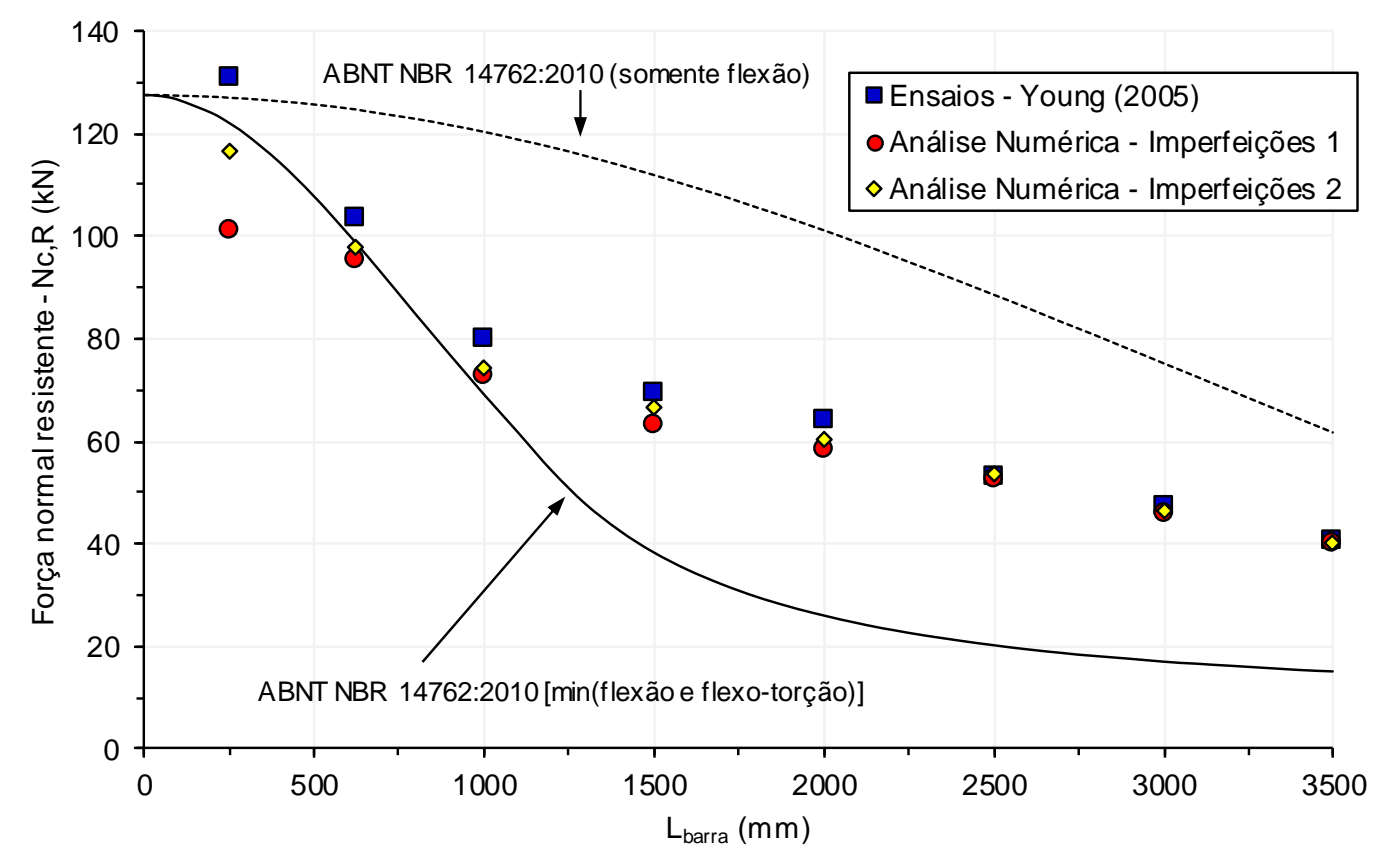

Figura 6 - Comparação dos resultados da análise numérica com os obtidos experimentalmente por Young (2005) e da ABNT NBR 14762:2010: seção Le 70x17×1,90
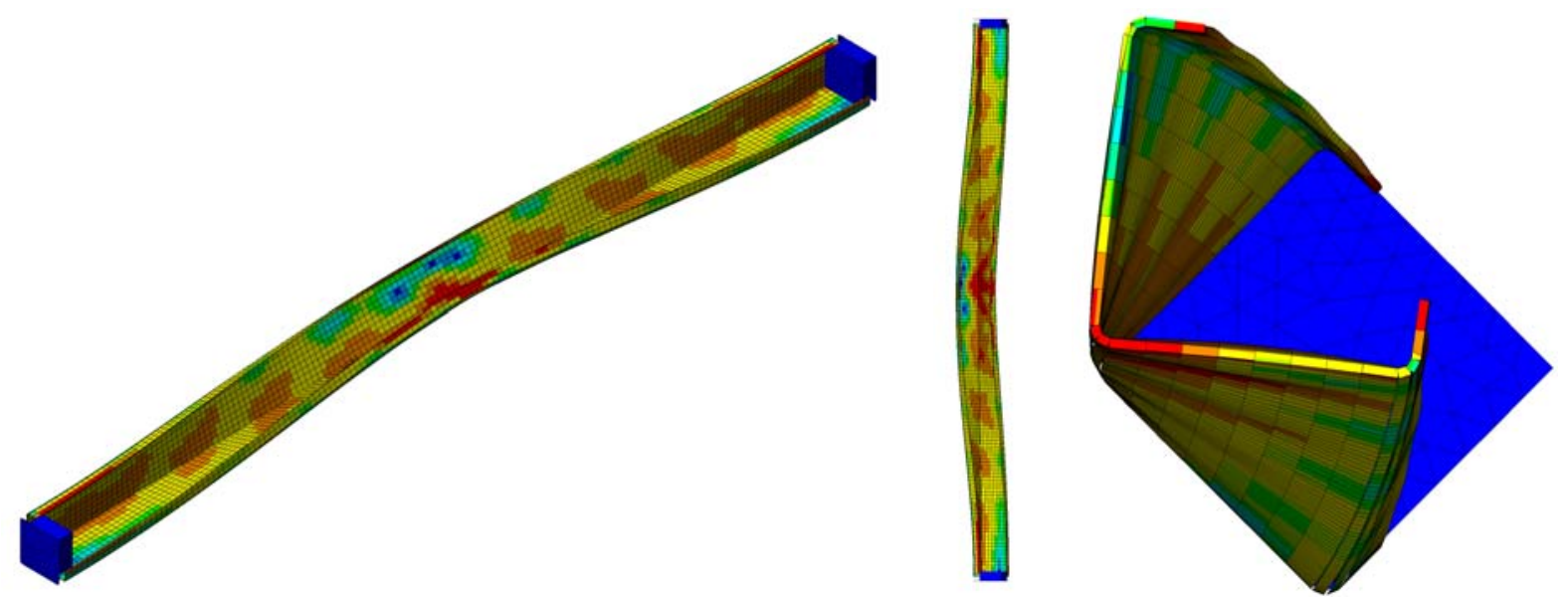

Figura 7 - Instabilidade local, por flexo-torção e flexão e distribuição de tensões de von Mises: (seção: Le 70x17x1,90, $L_{\text {barra }}=1500$ mm - Imperfeições: $L=0,14 t ; F T=0,64$ t e $F=L_{\text {barra }} / 1500$ )
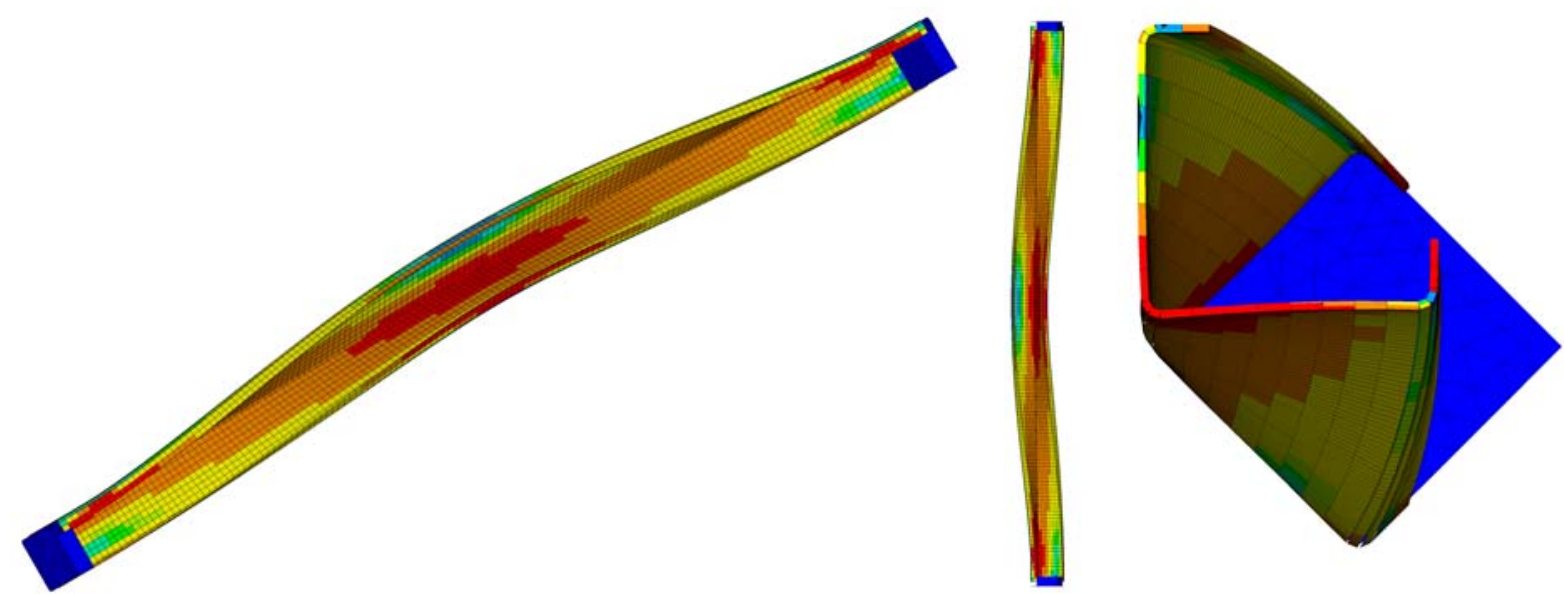

Figura 8 - Instabilidade por flexo-torção e flexão e distribuição de tensões de von Mises: (seção Le 70x17x1,90, Lbarra $=1500 \mathrm{~mm}$ - Imperfeições: $\mathrm{L}=0$; FT = 0,64t e F = Lbarra $/ 1500$ ) 
Comparando os resultados obtidos na análise via elementos finitos presente neste trabalho com os valores experimentais encontrados por Young (2005), observa-se que o modelo numérico é bastante satisfatório, pois tanto as barras em que foram inseridas todas as imperfeições (imperfeições 1) quanto aquelas em que pelo menos uma imperfeição não foi inserida (imperfeições 2) apresentaram força normal resistente muito próximas às obtidas nos ensaios experimentais.

No caso dos modos de falha observados, as barras em que pelo menos uma das imperfeições não foram inseridas (imperfeições 2) apresentaram maior similaridade em relação aos resultados experimentais. A escolha por incluir a imperfeição associada ao modo local na sobreposição das imperfeições produziu o modo de falha local em todas as barras, independente do seu comprimento e espessura. Este resultado não é totalmente coerente com o que foi encontrado experimentalmente por Young (2005).

No geral, os modos de instabilidade observados foram o local para barras mais curtas, flexo-torção para barras de comprimento intermediário e a combinação de flexotorção e flexão para barras mais longas. Cabe ressaltar que, para espessuras menores da seção $(1,20 \mathrm{~mm})$, o modo local aparece praticamente em todos os comprimentos.

\subsection{Cantoneira enrijecida submetida a compressão excêntrica}

Nas Tabelas 8, 9 e 10 e Figuras 9, 10 e 11 são apresentados os resultados da análise numérica das seções submetidas a compressão excêntrica comparados com as previsões teóricas calculadas de acordo com a ABNT NBR 14762:2010. Todas as simulações foram realizadas com a inserção de imperfeições de $0,14 \mathrm{t}$ para o modo local, 0,64t para o modo de flexo-torção e $L_{b a r r a} / 1500$ para o modo de flexão (imperfeições 1).

A comparação dos resultados obtidos na análise numérica por elementos finitos com os valores encontrados utilizando a ABNT NBR 14762:2010, demonstra que os procedimentos normativos são ainda contra a segurança em relação a barras curtas e conservadores em relação a barras mais esbeltas, mesmo não considerando o momento gerado pela excentricidade do carregamento. Quanto aos modos de falha, é possível observar similaridades entre a análise numérica e o procedimento normativo. A análise numérica apresenta predominantemente a sobreposição dos modos globais 
de flexão e flexo-torção para barras mais esbeltas e a sobreposição do modo local com o modo global de flexo-torção em barras curtas, estando relativamente de acordo com o que é previsto pela ABNT NBR 14762:2010.

Tabela 8 - Comparação dos resultados da análise numérica com os obtidos da ABNT NBR 14762:2010: seção Le 70x17x1,20

\begin{tabular}{|c|c|c|c|c|c|c|c|c|}
\hline $\begin{array}{l}\text { Lbarra } \\
(\mathrm{mm})\end{array}$ & $\begin{array}{c}N_{\mathrm{EF}} \\
(\mathrm{kN})\end{array}$ & $\begin{array}{l}\text { Modo de } \\
\text { Falha }\end{array}$ & $\begin{array}{l}N_{c, R}{ }^{1} \\
(k N)\end{array}$ & $\begin{array}{l}\text { Modo de } \\
\text { Falha }\end{array}$ & $\begin{array}{l}N_{c, R}{ }^{2} \\
\text { (kN) }\end{array}$ & $\begin{array}{c}\text { Modo de } \\
\text { Falha }\end{array}$ & $\mathbf{N}_{\mathrm{EF}} / \mathbf{N}_{\mathrm{C}, \mathrm{R}} \mathbf{R}$ & $\mathbf{N}_{\mathrm{EF}} / \mathbf{N}_{\mathrm{C}, \mathrm{R}}^{2}$ \\
\hline \multicolumn{9}{|c|}{ Extremidades dos dispositivos de apoio engastadas "perfis T" } \\
\hline \multicolumn{9}{|c|}{ Cantoneiras conectadas pela aba: $\mathrm{K}_{1}=\mathrm{K}_{2}=\mathrm{K}_{\mathrm{z}}=1,0$} \\
\hline 250 & 42,3 & $\mathrm{~L}$ & 60,8 & $\mathrm{~L}+\mathrm{FT}$ & 73,8 & $\mathrm{~L}+\mathrm{F}$ & 0,70 & 0,57 \\
\hline 1000 & 31,2 & $\mathrm{~L}+\mathrm{F}+\mathrm{FT}$ & 12,0 & FT & 57,0 & $L+F$ & 2,59 & 0,55 \\
\hline 1500 & 26,7 & $\mathrm{~F}+\mathrm{FT}$ & 6,8 & FT & 40,5 & $\mathrm{~L}+\mathrm{F}$ & 3,92 & 0,66 \\
\hline 2000 & 21,6 & $\mathrm{~F}+\mathrm{FT}$ & 5,0 & FT & 26,6 & $L+F$ & 4,35 & 0,81 \\
\hline 2500 & 17,0 & $\mathrm{~F}+\mathrm{FT}$ & 4,1 & FT & 18,7 & $L+F$ & 4,16 & 0,91 \\
\hline 3000 & 13,3 & $\mathrm{~F}+\mathrm{FT}$ & 3,6 & FT & 13,0 & $\mathrm{~F}$ & 3,68 & 1,02 \\
\hline 3500 & 10,6 & $\mathrm{~F}+\mathrm{FT}$ & 3,3 & FT & 9,6 & $\mathrm{~F}$ & 3,22 & 1,11 \\
\hline & & & & & \multicolumn{2}{|c|}{ Média } & 3,23 & 0,80 \\
\hline & & & & & \multicolumn{2}{|c|}{ CV } & 1,26 & 0,22 \\
\hline
\end{tabular}

Lbarra é o comprimento da barra.

$\mathrm{N}_{\mathrm{EF}}$ é a força normal excêntrica obtida por elementos finitos.

$\mathrm{L}=$ instabilidade local; $\mathrm{F}$ = instabilidade por flexão e FT = instabilidade por flexo-torção.

$C V$ = coeficiente de variação.

$\mathrm{N}_{\mathrm{c}, \mathrm{R}}{ }^{1}$ é calculado de acordo com a ABNT NBR 14762:2010 utilizando o mínimo entre flexão e flexotorção para o modo de instabilidade global (Procedimento recomendado).

$\mathrm{N}_{c, \mathrm{R}}{ }^{2}$ é calculado de acordo com a ABNT NBR 14762:2010 utilizando apenas o modo global de flexão.

Tabela 9 - Comparação dos resultados da análise numérica com os obtidos da ABNT NBR 14762:2010: seção Le 70×17×1,50

\begin{tabular}{|c|c|c|c|c|c|c|c|c|}
\hline $\begin{array}{l}\text { Lbarra } \\
(\mathrm{mm})\end{array}$ & $\begin{array}{l}N_{\mathrm{EF}} \\
(\mathrm{kN})\end{array}$ & $\begin{array}{l}\text { Modo de } \\
\text { Falha }\end{array}$ & $\begin{array}{l}N_{c, R}{ }^{1} \\
(k N)\end{array}$ & $\begin{array}{l}\text { Modo de } \\
\text { Falha }\end{array}$ & $\begin{array}{l}N_{c, R}{ }^{2} \\
(k N)\end{array}$ & $\begin{array}{l}\text { Modo de } \\
\text { Falha }\end{array}$ & $\mathrm{N}_{\mathrm{EF}} / \mathrm{N}_{\mathrm{c}, \mathrm{R}}{ }^{1}$ & $\mathbf{N}_{\mathrm{EF}} / \mathbf{N}_{\mathrm{c}, \mathrm{R}}{ }^{2}$ \\
\hline \multicolumn{9}{|c|}{$\begin{array}{l}\text { Extremidades dos dispositivos de apoio engastadas "perfis T" } \\
\text { Cantoneiras conectadas pela aba: } \mathrm{K}_{1}=\mathrm{K}_{2}=\mathrm{K}_{\mathrm{z}}=1,0\end{array}$} \\
\hline 250 & 54,5 & $\mathrm{~L}$ & 76,0 & $\mathrm{~L}+\mathrm{FT}$ & 90,5 & $L+F$ & 0,72 & 0,60 \\
\hline 625 & 47,9 & $\mathrm{~L}+\mathrm{F}+\mathrm{FT}$ & 33,5 & $\mathrm{~L}+\mathrm{FT}$ & 83,8 & $L+F$ & 1,43 & 0,57 \\
\hline 1000 & 40,6 & $\mathrm{~F}+\mathrm{FT}$ & 16,4 & $\mathrm{FT}$ & 72,6 & $L+F$ & 2,48 & 0,56 \\
\hline 1500 & 33,9 & $\mathrm{~F}+\mathrm{FT}$ & 10,1 & FT & 54,0 & $L+F$ & 3,36 & 0,63 \\
\hline 2000 & 26,1 & $\mathrm{~F}+\mathrm{FT}$ & 7,9 & FT & 36,0 & $L+F$ & 3,32 & 0,72 \\
\hline 2500 & 20,2 & $\mathrm{~F}+\mathrm{FT}$ & 6,8 & FT & 23,9 & $\mathrm{~F}$ & 2,98 & 0,85 \\
\hline 3000 & 16,0 & $\mathrm{~F}+\mathrm{FT}$ & 6,2 & FT & 16,6 & $\mathrm{~F}$ & 2,59 & 0,96 \\
\hline \multirow[t]{3}{*}{3500} & 12,8 & $\mathrm{~F}+\mathrm{FT}$ & 5,7 & FT & 12,2 & $F$ & 2,24 & 1,05 \\
\hline & & & & & \multicolumn{2}{|c|}{ Média } & 2,39 & 0,70 \\
\hline & & & & & \multicolumn{2}{|r|}{ CV } & 0,92 & 0,19 \\
\hline
\end{tabular}

$L_{\text {barra }}$ é o comprimento da barra.

$N_{\mathrm{EF}}$ é a força normal excêntrica obtida por elementos finitos.

$\mathrm{L}=$ instabilidade local; $\mathrm{F}$ = instabilidade por flexão e $\mathrm{FT}=$ instabilidade por flexo-torção.

$\mathrm{CV}=$ coeficiente de variação.

$\mathrm{N}_{c, R^{1}}$ é calculado de acordo com a ABNT NBR 14762:2010 utilizando o mínimo entre flexão e flexotorção para o modo de instabilidade global (Procedimento recomendado).

$\mathrm{N}_{c, R}{ }^{2}$ é calculado de acordo com a ABNT NBR 14762:2010 utilizando apenas o modo global de flexão. 
Tabela 10 - Comparação dos resultados da análise numérica com os obtidos da ABNT NBR 14762:2010: seção Le 70x17x1,90

\begin{tabular}{|c|c|c|c|c|c|c|c|c|}
\hline $\begin{array}{l}\mathrm{L}_{\text {barra }} \\
(\mathrm{mm})\end{array}$ & $\begin{array}{l}N_{\mathrm{EF}} \\
(\mathrm{kN})\end{array}$ & $\begin{array}{l}\text { Modo de } \\
\text { Falha }\end{array}$ & $\begin{array}{l}N_{c, R}{ }^{1} \\
(k N)\end{array}$ & $\begin{array}{l}\text { Modo de } \\
\text { Falha }\end{array}$ & $\begin{array}{l}N_{c, R}{ }^{2} \\
(k N)\end{array}$ & $\begin{array}{l}\text { Modo de } \\
\text { Falha }\end{array}$ & $\mathbf{N}_{\mathrm{EF}} / \mathbf{N}_{\mathrm{C}, \mathrm{R}^{1}}{ }^{1}$ & $\mathrm{~N}_{\mathrm{EF}} / \mathrm{N}_{\mathrm{c}, \mathrm{R}}{ }^{2}$ \\
\hline \multicolumn{9}{|c|}{$\begin{array}{l}\text { Extremidades dos dispositivos de apoio engastadas "perfis T" } \\
\text { Cantoneiras conectadas pela aba: } \mathrm{K}_{1}=\mathrm{K}_{2}=\mathrm{K}_{2}=1,0\end{array}$} \\
\hline 250 & 81,6 & $\mathrm{~L}+\mathrm{FT}$ & 107,7 & $\mathrm{~L}+\mathrm{FT}$ & 125,5 & $L+F$ & 0,76 & 0,65 \\
\hline 625 & 66,3 & $\mathrm{~F}+\mathrm{FT}$ & 50,9 & $\mathrm{FT}$ & 116,3 & $L+F$ & 1,30 & 0,57 \\
\hline 1000 & 55,3 & $\mathrm{~F}+\mathrm{FT}$ & 26,1 & FT & 101,1 & $L+F$ & 2,12 & 0,55 \\
\hline 1500 & 42,4 & $\mathrm{~F}+\mathrm{FT}$ & 17,1 & FT & 75,3 & $L+F$ & 2,47 & 0,56 \\
\hline 2000 & 32,7 & $\mathrm{~F}+\mathrm{FT}$ & 13,9 & FT & 47,9 & $\mathrm{~F}$ & 2,35 & 0,68 \\
\hline 2500 & 25,4 & $\mathrm{~F}+\mathrm{FT}$ & 12,3 & FT & 30,7 & $\mathrm{~F}$ & 2,06 & 0,83 \\
\hline 3000 & 20,1 & $\mathrm{~F}+\mathrm{FT}$ & 11,3 & FT & 21,3 & $\mathrm{~F}$ & 1,78 & 0,94 \\
\hline \multirow[t]{3}{*}{3500} & 16,1 & $\mathrm{~F}+\mathrm{FT}$ & 10,6 & FT & 15,7 & $\mathrm{~F}$ & 1,53 & 1,03 \\
\hline & & & & & \multicolumn{2}{|c|}{ Média } & 1,80 & 0,68 \\
\hline & & & & & \multicolumn{2}{|c|}{ CV } & 0,58 & 0,19 \\
\hline
\end{tabular}

Lbarra é o comprimento da barra.

$\mathrm{N}_{\mathrm{EF}}$ é a força normal excêntrica obtida por elementos finitos.

$\mathrm{L}=$ instabilidade local; $\mathrm{F}$ = instabilidade por flexão e FT = instabilidade por flexo-torção.

$\mathrm{CV}=$ coeficiente de variação.

$\mathrm{N}_{\mathrm{C}, \mathrm{R}}{ }^{1}$ é calculado de acordo com a ABNT NBR 14762:2010 utilizando o mínimo entre flexão e flexotorção para o modo de instabilidade global (Procedimento recomendado).

$\mathrm{N}_{\mathrm{c}, \mathrm{R}}{ }^{2}$ é calculado de acordo com a ABNT NBR 14762:2010 utilizando apenas o modo global de flexão.

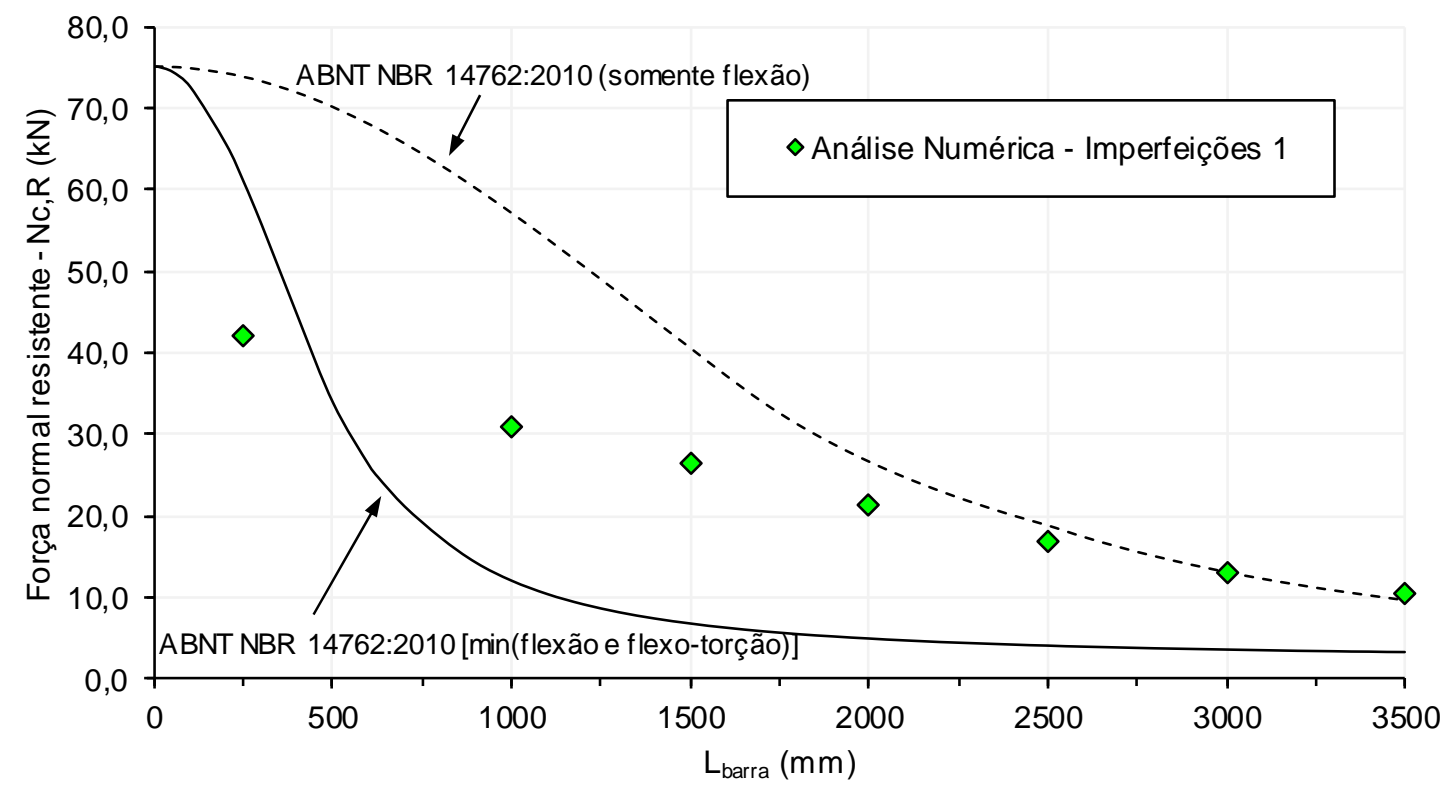

Figura 9 - Comparação dos resultados da análise numérica com os obtidos da ABNT NBR 14762:2010: seção Le $70 \times 17 \times 1,20$ 


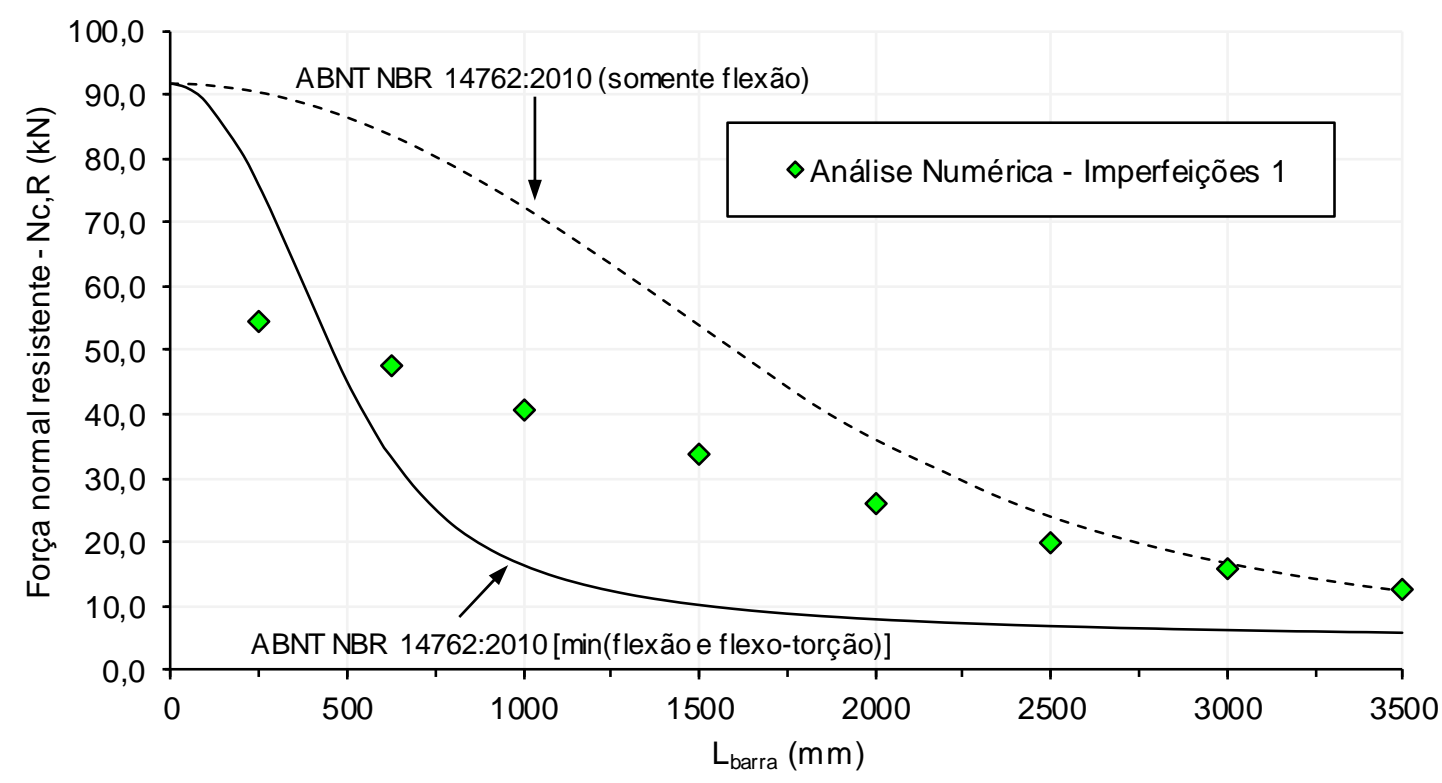

Figura 10 - Comparação dos resultados da análise numérica com os obtidos da ABNT NBR 14762:2010: Le 70x17x1,50

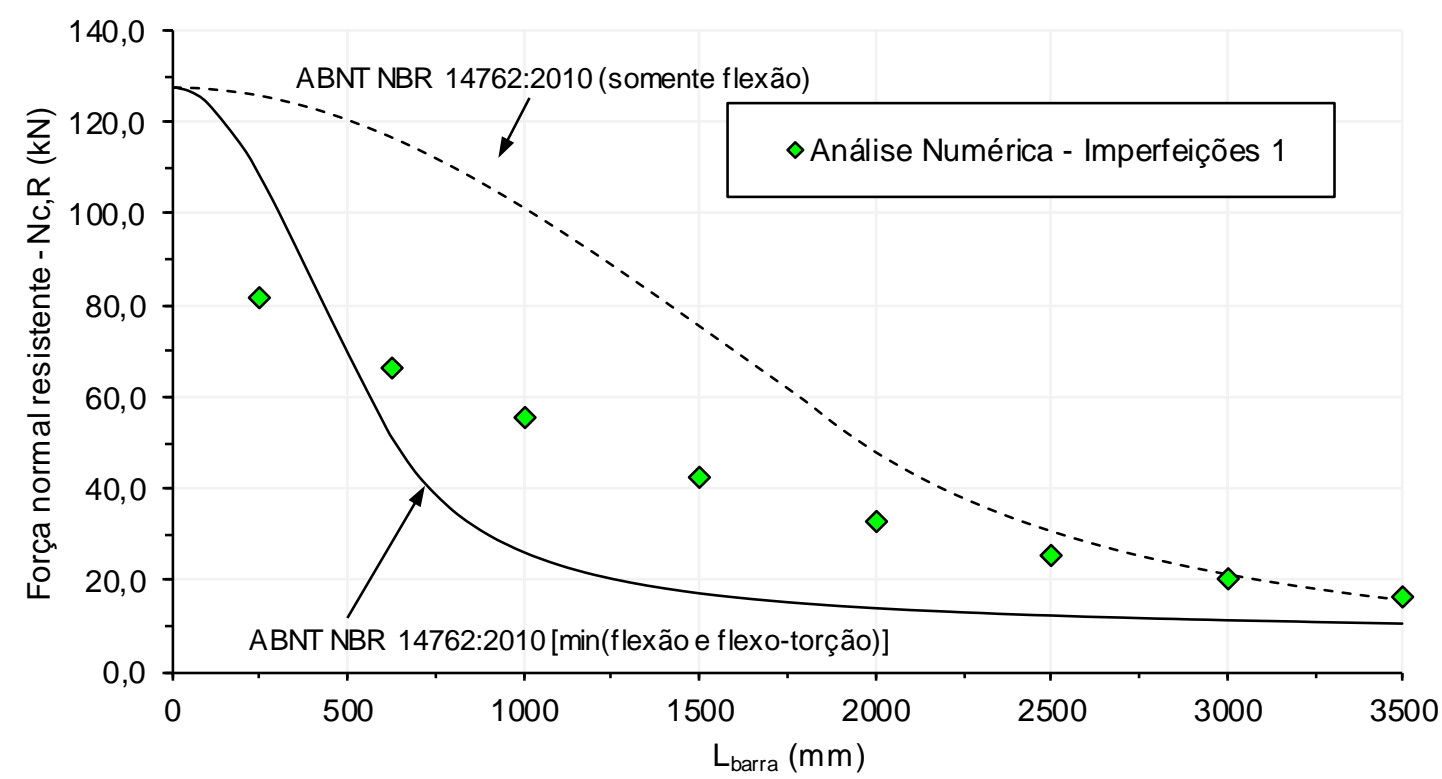

Figura 11 - Comparação dos resultados da análise numérica com os obtidos da ABNT NBR 14762:2010: seção: Le 70×17×1,90

Na Figura 12 são apresentados típicos modos de instabilidade observados na análise numérica de cantoneiras conectadas pela aba. As mesmas figuras ilustram o panorama de tensões de von Mises observado no instante em que a barra atinge a resistência máxima. 

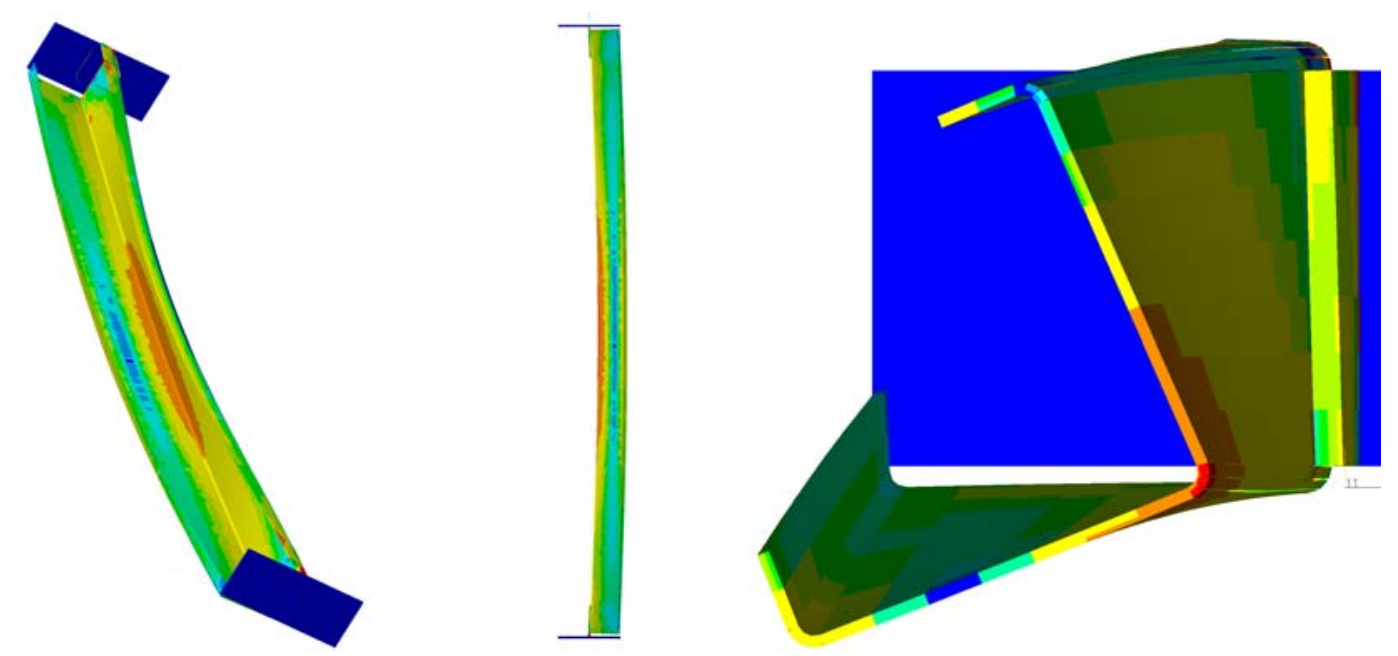

Figura 12 - Instabilidade por flexo-torção e flexão e distribuição de tensões de von Mises: (seção: Le 70x17x1,90, Lbarra $=1500 \mathrm{~mm}$ - Imperfeições: $L=0,14 t ; F T=0,64 t$ e $F=L_{\text {barra }} / 1500$ )

\section{Conclusões}

As análises via elementos finitos, para as cantoneiras submetidas a compressão centrada, apresentaram valores relativamente próximos aos obtidos na análise experimental apresentada por Young (2005). A consideração e desconsideração de imperfeições geométricas iniciais locais (Imperfeições 2) acarretaram em resultados mais condizentes com a realidade, demonstrando a significativa sensibilidade das cantoneiras quanto as imperfeições geométricas locais.

Para as cantoneiras submetidas a força de compressão centrada e excêntrica, a ABNT NBR 14762:2010 se mostrou conservadora aos resultados via elementos finitos, exceto para barras curtas. Faz-se necessário, deste modo, uma revisão dos procedimentos de cálculo a serem seguidos. Com relação à hipótese de cálculo adotada, considerando para o modo global apenas flexão, a mesma se mostrou contra a segurança em praticamente todos os casos analisados para compressão centrada e excêntrica.

O uso do software Ansys ${ }^{\circledR}$ permitiu estudar os modelos ensaiados por Young (2005) e comparar com os dimensionados pela ABNR NBR 14762:2010 de forma eficaz, apesar da grande sensibilidade apresentada pelos perfis formados a frio quanto a imperfeições geométricas iniciais.

Portanto, este trabalho demonstra a validade dos resultados obtidos, tendo em vista aumentar o conhecimento sobre perfis formados a frio, com seção de cantoneira enrijecida, submetida a compressão centrada e excêntrica. 


\section{Agradecimentos}

Os autores agradecem ao CNPq - Conselho Nacional de Desenvolvimento Científico e Tecnológico pelo apoio da pesquisa.

\section{Referências bibliográficas}

ALMEIDA, S. J. C. Análise numérica de perfis de aço formados a frio comprimidos considerando imperfeições geométricas iniciais. Dissertação de Mestrado - Escola de Engenharia de São Carlos, Universidade de São Paulo, São Carlos, 2007.

ANSYS. Structural nonlinearities. v.13.0, Houston, USA, 2011.

ASSOCIAÇÃO BRASILEIRA DE NORMAS TÉCNICAS - ABNT. NBR 14762:2010. Dimensionamento de estruturas de aço constituídas por perfis formados a frio. Rio de Janeiro, 2010.

CHAJES, A.; WINTER, G. Torsional-flexural buckling of thin-walled members. Journal of the Structural Division, ASCE, v. 91, 1965.

CHODRAUI, G. M. B. Análise teórica e experimental de perfis de aço formados a frio submetidos à compressão. Tese de Doutorado - Escola de Engenharia de São Carlos Universidade de São Paulo, São Carlos, 2006.

LOURENÇO, P. B. Métodos computacionais na mecânica dos sólidos não linear. Relatório 99-DEC/E-1, Departamento de Engenharia Civil Universidade do Minho, Braga, Portugal, 1999.

MAIA, W. F. Sobre a estabilidade de cantoneiras de aço formadas a frio submetidas à compressão. Dissertação (Mestrado) - Escola de Engenharia de São Carlos, Universidade de São Paulo, São Carlos, 2008.

SCHAFER, B. W., PEKÖZ, T. Computational modeling of cold-formed steel: characterizing geometric imperfections and residual stresses. Journal of Constructional Steel Research, v.47, 193-210, 1998.

SHIFFERAW, Y.; SCHAFER, B. W. Cold-formed steel lipped and plain angle columns with fixed ends. Thin-Walled Structures, v. 80, p. 142-152, 2014.

SHIFFERAW, Y.; SCHAFER, B. W. Behavior and design of cold-formed steel lipped and plain angles. Structural Stability Research Council Annual Stability Conference. ASC. P. 260-270, May 10-14, Pittsburgh, PA, USA, 2011.

TIMOSHENKO, S. P.; GERE, J. M. Theory of elastic stability. 2. ed. New York: McGrawHill. 541p, 1961.

WINTER, G. Strength of Thin Steel Compression Flanges. Transactions ASCE, Vol. 112, Issue 1, Pg. 527-554, 1947.

YOUNG, B. Experimental investigation of cold-formed steel lipped angle concentrically loaded compression members. Journal of Structural Engineering, v. 131, n. 9, p. 13901396, 2005. 